\title{
Measuring originality in science
}

\author{
Sotaro Shibayama ${ }^{1}$ (D) Jian Wang ${ }^{2}$
}

Received: 29 March 2019 / Published online: 11 November 2019

(c) The Author(s) 2019

\begin{abstract}
Originality has self-evident importance for science, but objectively measuring originality poses a formidable challenge. We conceptualise originality as the degree to which a scientific discovery provides subsequent studies with unique knowledge that is not available from previous studies. Accordingly, we operationalise a new measure of originality for individual scientific papers building on the network betweenness centrality concept. Specifically, we measure the originality of a paper based on the directed citation network between its references and the subsequent papers citing it. We demonstrate the validity of this measure using survey information. In particular, we find that the proposed measure is positively correlated with the self-assessed theoretical originality but not with the methodological originality. We also find that originality can be reliably measured with only a small number of subsequent citing papers, which lowers computational cost and contributes to practical utility. The measure also predicts future citations, further confirming its validity. We further characterise the measure to guide its future use.
\end{abstract}

Keywords Scientific originality $\cdot$ Novelty $\cdot$ Network centrality $\cdot$ Citation network

\section{Introduction}

As science progresses through discoveries of new knowledge, originality constitutes one of the core values in science (Gaston 1973; Hagstrom 1974; Merton 1973; Storer 1966). As such, originality is highly regarded in the recognition system of science and is relevant for critical science decisions such as funding allocation, hiring, tenure evaluation, and scientific awards (Dasgupta and David 1994; Merton 1973; Stephan 1996; Storer 1966). Despite its importance, originality of scientific discoveries is hard to measure. In practice, originality is often evaluated by means of peer reviews (Chubin and Hackett 1990), which is feasible only in a small scale, whereas assessing originality in a large scale poses a formidable

Electronic supplementary material The online version of this article (https://doi.org/10.1007/s 1119 2-019-03263-0) contains supplementary material, which is available to authorized users.

Sotaro Shibayama

sotaro.shibayama@fek.lu.se

1 School of Economics and Management, Lund University, 22363 Lund, Sweden

2 Leiden University, 2333 CA Leiden, The Netherlands 
challenge. Though bibliometric studies have recently made a considerable advancement in measuring various aspects of scientific discoveries (Boudreau et al. 2016; Foster et al. 2015; Lee et al. 2015; Trapido 2015; Uzzi et al. 2013; Wang et al. 2017), originality per se has rarely been measured. This study proposes a new measure of originality building on the network betweenness centrality concept (Borgatti and Everett 2006; Freeman 1979) and measures the originality of a scientific paper based on the directed citation network between its references and subsequent papers citing the focal paper. To validate the proposed originality measure, we conducted a questionnaire survey and demonstrate that the proposed measure is significantly correlated with the self-assessed theoretical originality but not with the methodological originality. The result also shows that the measure can predict the number of citations that the focal paper receives in the future, which further confirms the validity of the measure. We also find that the originality can be reliably measured with only a small number of subsequent citing papers. This substantially lowers computational cost compared to previous related bibliometric measures and facilitates practical use of the measure.

\section{Literature review}

Though originality is one of the core values in science, there is not yet a clear consensus on what it exactly means (Dirk 1999; Guetzkow et al. 2004). In a broad sense, originality could mean anything new (e.g., new method, new theory, and new observation) that adds to the common stock of scientific knowledge. To differentiate the degree of newness, the sociology of science literature has argued that scientific discoveries can either conform to the tradition or depart from it, and only the latter is considered to be original (Bourdieu 1975; Kuhn 1970). Following this stream of thoughts, we define originality as the degree to which a scientific discovery provides subsequent studies with unique knowledge that is not available from previous studies. As further explained, this definition is in line with the network betweenness centrality concept and allows a straightforward operationalisation.

\section{Existing measures of originality}

Despite the theoretical interest in and practical relevance of originality, how to measure originality is under-developed. In small scales, a few studies explored the aspects in which research must be new in order to be perceived by scientists as original (Dirk 1999; Guetzkow et al. 2004). Dirk (1999) conducted a questionnaire survey to evaluate three dimensions (hypotheses, methods, and results) of newness, finding that life scientists consider research with unreported hypotheses as original rather than research with new methods. Through in-depth interviews of social scientists, Guetzkow et al. (2004) also identified various dimensions of newness associated with perceived originality: approach, theory, method, data, and findings. They also found that relevant dimensions of originality can differ across scientific fields.

Bibliometric techniques for science decisions have been rapidly developing thanks to the advanced computing power and enriched bibliometric data (Hicks et al. 2015). A few approaches to measure the newness (originality, novelty, creativity, etc.) of a study are worth noting, although they are not necessarily labelled as "originality." The first approach considers originality as a quality established only through reuse of a study by subsequent studies or the collective evaluation by peer scientists, but not as an intrinsic quality of a 
study (Merton 1973). For example, Wang (2016), following the definition of creativity (Amabile 1983), argues that forward citation counts can be viewed as peer recognition of novelty and usefulness, and therefore is a proxy for creativity.

The second approach is more recently developed and views originality as an inherent quality of a scientific paper that can be measured at the time of publication, irrespective of subsequent use of the paper. This approach has several nuanced conceptualisation strategies. One strategy focuses on the newness of a study based on the introduction of a new concept or object. For example, Azoulay et al. (2011) measured the novelty of an article based on the age of keywords assigned to the article. Within the field of biochemistry, Foster et al. (2015) also measured the novelty on the basis of new chemical entities introduced in a study. This approach is intuitively straightforward but requires a reliable and up-to-date dictionary encoding all existing concepts and objects, which is not always the case.

Another strategy is based on the assumption that integrating a broader scope of knowledge is a sign of newness. For example, the originality of a patent is operationalised as the diversity of technological domains it cites, where diversity is measured using the Herfindahl-type index of patent classes that the focal patent cite (Hall et al. 2001; Harrigan et al. 2017; Trajtenberg et al. 1997). A similar approach is used to measure the interdisciplinarity of scientific papers (Stirling 2007; Wang et al. 2015; Yegros-Yegros et al. 2015), though it conceptually features diversity rather than originality.

A third strategy, building on the combinatorial novelty perspective, views novelty as making new or unusual combinations of pre-existing knowledge components, where knowledge components can be operationalised by keywords (Boudreau et al. 2016), referenced articles (Trapido 2015), referenced journals (Uzzi et al. 2013; Wang et al. 2017), and chemical entities (Foster et al. 2015). An obvious limitation of this approach is that it captures only the combinatorial novelty but not other types of novelty.

Our proposed conceptualisation of originality lies between these two approaches. We consider originality to be rooted in a set of information included in a focal scientific paper. However, we argue that the value of the paper is realised through its reuse by other scientists, and that its originality is established through its interaction with other scientists and follow-on research (Latour and Woolgar 1979; Merton 1973; Whitley 1984). A few recently developed measures, though not conceptualised as originality, are in line with this approach. For example, Funk and Owen-Smith (2017) assess whether an invention destabilises or consolidates existing technology streams, by examining the pattern of forward citations to a focal patent and its references. This measure is adopted by Wu et al. (2019) to evaluate the disruptiveness of scientific papers. Similarly, Bu et al. (2019) measure the independent impact of papers based on the co-citation and bibliographic coupling between a focal paper and its citing papers.

\section{Proposed measure of originality}

\section{Base measure}

We propose to measure the originality of an individual scientific papers based on its cited papers (i.e., references) and citing papers (i.e. follow-on research). We draw on subsequent papers that cite the focal paper to evaluate whether the authors of these subsequent citing papers perceive the focal paper as an original source of knowledge (Fig. 1A). Suppose that the focal paper $X$ cites a set of prior papers (reference set $R$ ) and is cited by a set of subsequent 


\section{(A) Base network}

$\mathrm{N}$ papers cited by $\mathrm{X}$

(Reference set R)

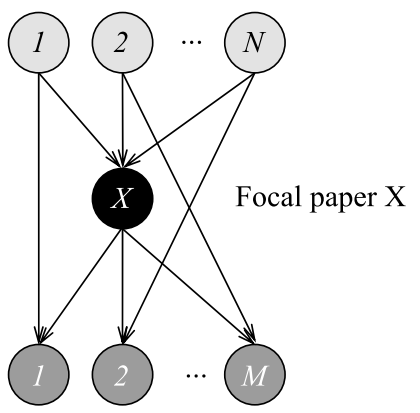

$\mathrm{M}$ papers citing $\mathrm{X}$

(Citing set $\mathrm{C}$ )

\section{(B) Extended network}

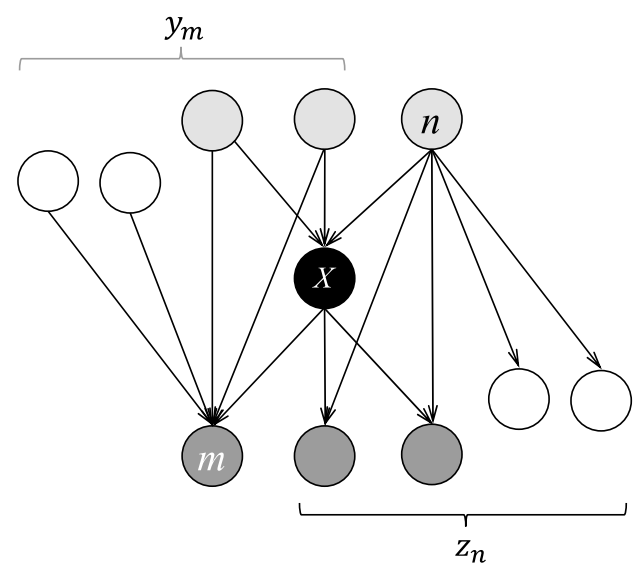

Fig. 1 Directed network of papers and citations. Note: Papers are the nodes, and citation links are the directional edges, where nodes with arrowheads cite nodes with arrow tails

papers ( citing set $C$ ). If $X$ serves as a more original source of knowledge, then the citing papers (i.e., papers in citing set $C$ ) are less likely to rely on papers that are cited by $X$ (i.e., papers in reference set $R$ ). In contrast, if $X$ is not original but an extension of $R$, then $C$ will probably also cite $R$ together with $X$. In other words, we exploit the evaluation by the authors of followon research to measure the originality of the focal paper.

This idea is operationalised as follows. Suppose that the focal paper $X$ cites $N$ references and is cited by $M$ subsequent papers. For the $n$-th reference $(n \in\{1, \ldots, N\})$ and the $m$-th citing subsequent paper $(m \in\{1, \ldots, M\})$, define $x_{n m}$ as follows:

$$
x_{n m}= \begin{cases}1 & \text { if } m \text { cites } n \\ 0 & \text { otherwise }\end{cases}
$$

In Fig. 1A, for example, $x_{11}=1$ and $x_{12}=0$. If a subsequent citing paper $m$ cites few references of $X$, it implies that $X$ provides original knowledge for $m$ that is not provided by reference set $R$. In contrast, if the subsequent citing paper $m$ cites many references of $X$, it implies that the author of $m$ perceives the focal study $X$ as being unoriginal. Thus, the originality score of the focal study $X$ evaluated by the author of $m$ is the share of papers cited both by $X$ and by $m$ :

$$
1-\frac{1}{N} \sum_{n=1}^{N} x_{n m}
$$

This calculation is repeated for $M$ citing papers, and the mean value is used as the originality score for $X$ :

$$
\operatorname{Orig}_{\text {base }}=1-\frac{1}{M N} \sum_{m=1}^{M} \sum_{n=1}^{N} x_{n m}
$$


This measure corresponds to the proportion of 0's in the citation matrix (i.e., missing citation links) between the cited and citing papers of $X$. This measure ranges from 0 to 1 , and a higher value implies a higher level of originality.

To add a theoretical basis to the proposed measure, we draw on the network centrality framework (Borgatti and Everett 2006; Freeman 1979). In short, Eq. (3) is equivalent to the normalised betweenness centrality of $X$ in the directed citation network (see Appendix 1). Betweenness centrality is defined as the number of the shortest paths that pass through the focal node among every pair of nodes in a connected network (Freeman 1979). Betweenness centrality has been used as a measure of mediation and brokerage in various networks, such as transportation flow and employee interaction in organisations (Flynn and Wiltermuth 2010; Gomez et al. 2013; Puzis et al. 2013). The intuition is that a directed network represents the flow of information from origins (e.g., cited papers) to destinations (e.g., citing papers), and that a node with a high level of betweenness centrality plays an important intermediary role in passing information in the network. This is consistent with the derivation of our measure. High values in the proposed originality measure indicate that the focal paper $X$ cannot be bypassed in the flow of knowledge from old studies to recent studies; in other words, $X$ provides original knowledge.

Prior bibliometric studies have used betweenness centrality to analyse citation networks, but most studies have treated citation networks as undirected. Because the information of citation direction is lost, the interpretation of betweenness centrality has been concerned more with connectedness than with information flow (Leydesdorff 2007). Previous studies have also found that papers with high betweenness centrality tend to receive more citations in the future (Shibata et al. 2007; Topirceanu et al. 2018).

Note that the proposed measure restricts the scope of network to the immediate neighbours of a focal node, whereas most previous studies draw on the whole network. Although this constraint overlooks information about remote nodes and links, using the whole network is not without limitation. Importantly, the computation with the whole network causes "double counting" because many paths between remote nodes can share the same subsets of links (Borgatti and Everett 2006; Brandes 2008). It also incurs substantial computational burden especially for large networks. A few variant betweenness centrality operationalisations have been proposed to address these issues (Borgatti and Everett 2006). Among others, $k$-betweenness centrality (or bounded-distance betweenness centrality) considers only paths with length of $k$ or shorter, where $k$ is a positive integer (Freeman et al. 1991). Two-betweenness centrality is a special case, which considers only immediate neighbours to focal nodes (Gould and Fernandez 1989), as our proposed measures do. Previous studies have found that $k$-betweenness centrality with small $k$ 's can reasonably predict the betweenness centrality of the whole network (Ercsey-Ravasz et al. 2012), while it substantially reduces the computational cost.

\section{Weighted measure}

A potential weakness of the base measure in Eq. (3) is that it can be biased by the number of references cited by papers in citing set $C$, as well as the number of citations received by papers in reference set $R$. Namely, subsequent papers with many references are more likely to cite papers in reference set $R$, and highly cited papers in reference set $R$ are more likely to be cited by subsequent papers in citing set $C$ (Fig. 1B). To correct these potential biases, we propose Eqs. (4) and (5) as weighted measures:

$$
\text { Orig }_{\text {weighted1 }}=1-\frac{L}{N} \frac{\sum_{m=1}^{M} \sum_{n=1}^{N} x_{n m}}{\sum_{m=1}^{M} y_{m}}
$$




$$
\text { Orig }_{\text {weighted } 2}=1-L \frac{\sum_{m=1}^{M} \sum_{n=1}^{N} x_{n m}}{\sum_{m=1}^{M} y_{m} \sum_{n=1}^{N} z_{n}}
$$

where $y_{m}$ is the reference count of the $m$-th paper in citing set $C, z_{n}$ is the citation count of the $n$-th paper in reference set $R$, and $L$ is an arbitrary positive number. Appendix 2 further explains the derivation of the weighted measures.

\section{Use of citations for bibliometric measures}

Our proposed operationalisation strategy for originality is based on citation links between scientific papers. Although citation network has been widely used for science studies and research evaluations (Garfield 1955; Hicks et al. 2015; Martin and Irvine 1983; Uzzi et al. 2013), a few potential limitations are worth noting. In particular, though one paper citing another paper is supposed to indicate an intellectual connection between them (Garfield 1955; Small 1978), citations may embody different information. For example, citing papers may be considerably influenced by citing papers but may cite them only casually; citing papers may be built on cited papers but may disprove them (Bornmann and Daniel 2008; De Bellis 2009); and citations may be generated for social and political motivations rather than for intellectual reasons (De Bellis 2009; Gilbert 1977).

One important complication pertains to field differences. For example, some disciplines (e.g., mathematics) have less citing papers or shorter reference lists (Moed et al. 1985); some disciplines (e.g., social sciences) tend to cite older papers than others (e.g., natural sciences) (Price 1986); and the citation accumulation process is slower in some fields (e.g., social sciences and mathematics) than others (e.g., medical and chemistry) (Glänzel and Schoepflin 1995). As later discussed, these differences might call for adjustment in the scope of the citing set and the weighting, even though the generic framework applies to all fields.

\section{Methods}

\section{Sample}

For assessing the criterion validity of our originality measure for individual papers, we conducted a questionnaire survey of the authors of these papers to enquire into self-assessed originality. We selected a sample of active scientists who earned their $\mathrm{PhD}$ degrees in the field of life sciences in 1996-2011 in Japan for the following reasons. First, we need articles published several years ago (but not too recently) to compute our originality measure based on their forward citations. Second, we focus on papers from $\mathrm{PhD}$ dissertation projects, which are usually the first research project that scientists engage in and could help their recollection. We also expect that the respondents' desirability bias in evaluating originality could be mitigated since dissertation projects are usually decided by supervisors rather than respondents themselves. Finally, we focus on a single field of life sciences to rule out the heterogeneity across different scientific disciplines. ${ }^{1}$

\footnotetext{
1 Note that our validity exercise is made only in the field of life sciences. Since the citation cycle can differ between fields, future research should assess the validity of our measure in other disciplines.
} 
We randomly chose 573 scientists who meet the following conditions: (1) the information of $\mathrm{PhD}$ degree is publicly available through online dissertation databases, (2) the $\mathrm{PhD}$ dissertation projects were in the field of life sciences according to the funding information, and (3) the scientists remain in academic careers as of 2018. We mailed a survey to the scientists and collected 268 responses (response rate $=47 \%$ ). As 22 respondents had no papers during the $\mathrm{PhD}$ period, we used remaining 246 scientists as the main sample.

\section{Self-assessed measures of originality}

The respondents of the survey were asked to evaluate their own dissertation projects in two dimensions of originality: theoretical and methodological (Dirk 1999). Each dimension is measured in a three-point scale-0: not original (all or most of the theories/methods had already been reported in prior literature, or the project did not aim at the originality in the dimension), (1) somewhat original (part of the theories/methods had already been reported in prior literature, and (2) original (the theories/methods had not been reported in prior literature).

\section{Computing proposed originality measures}

We selected 564 papers that the respondents published as the first or second author in the year of their graduation or 1-2 year before. We exclude papers published after graduation because they can be either from $\mathrm{PhD}$ dissertation or from postdoc research, which may confound our analysis. From Web of Science (WoS), we obtained the bibliometric information of the focal papers, their references, and subsequent citing papers up to 2018. We then identified all citation links between the references and citing papers.

In computing the originality measures, the scope of citing set $C$ can be arbitrarily chosen. For example, it may include all existing citing papers to date or may be a single citing paper. Since the choice of citing sets can influence the quality of the measurement as well as the computational cost, we prepare two series of citing sets and assess their validities. The first series is based on the publication year of citing papers. The citing set $C^{(t)}$ includes citing papers published within $t$ years after the publication of the focal paper $(t=1,2, \ldots)$. For example, $C^{(3)}$ includes citing papers published in the same year as the focal paper or 1-3 years after that. Note that the size of $C^{(t)}$ can differ between focal papers when they have different forward citation counts. The second series of citing sets control for this variation. The citing set $C^{[s]}$ consists of the first $s$ citing papers $(s=1,2, \ldots)$. In order to control for the timing of the publication of citing papers, we include citing papers published only within three years after the focal paper. ${ }^{2}$

The second weighted measure (Eq. 5) uses the forward citation count of each cited paper $\left(z_{n}\right)$ in reference set $R$. Here, the time-window of the forward citation can be also arbitrarily chosen. In this validation exercise, we use the one-year period after the publication of the focal paper as the citation time-window.

\footnotetext{
${ }^{2}$ This time-window is chosen because our evaluation of $C^{(t)}$ suggests that citations only in the first few years are informative (see the next section). The measure is not calculated if a focal paper received fewer than $s$ citing papers within 3 years.
} 
As above discussed, citation accumulation process differs across scientific fields. Thus, the optimal choice of the citing set (parameters $t$ and $s$ ) as well as the citation time-window for the forward citation to the reference set should be identified in respective fields.

\section{Predicting future citations}

We also examine whether the proposed measure can predict future citation impact for assessing the construct validity of our proposed originality measure (Babbie 2012). Specifically, we use a dummy variable (Top10) as the dependent variable, coded 1 if a paper is among the top $10 \%$ highly-cited as of 2018 in the same cohort of papers with the same publication year and in the same field, and 0 otherwise.

\section{Results}

\section{Correlation with self-assessed originality}

First, we establish the criterion-related validity of the proposed originality measure (Babbie 2012). From randomly selected 246 scientists, we obtained the information of self-assessed originality of their past papers by a questionnaire survey. For potential multi-dimensionality of the originality concept, we measured two dimensions of originality: theoretical and methodological (see Appendix 3 for the distribution of the measures) (Dirk 1999). Then, we computed the proposed originality measures for 564 journal articles published by the survey respondents ${ }^{3}$ to analyse the correlations with the survey measures.

Because the scope of citing set $C$ can influence the quality of the measurement, we assess the validity of a series of citing sets. We first calculate the originality scores using citing papers published within the first $t$ years after the focal paper $(t=1, \ldots, 6)$. For each pair between the two survey measures (theory and method) and the three bibliometric measures (base and two weighted measures) with different citing sets, Fig. 2A shows the correlation coefficients. We observe that our proposed originality measures have significantly positive correlations with the survey measure of theoretical originality (for example, $r_{\text {base }}=0.130, p<0.05 ; r_{\text {weighted } 1}=0.136, p<0.001 ; r_{\text {weighted } 2}=0.142, p<0.001$ at $t=1$ ) but not with the methodological originality $(p>0.1)$. Provided that the previous literature found that life scientists tended to perceive theoretical newness, but not methodological newness, as relevant for originality (Dirk 1999), our proposed measures appear to capture the relevant dimension of originality. As to the size of the citing sets, the result indicates that the correlation with self-assessed theoretical originality is significant by using the citing papers only in the first year. A slight increase of the correlation coefficients is observed by using a larger citing sets $(t=3)$, but too large citing sets do not improve the correlation. This result suggests using citing papers only in the first or a few years for assessing originality, especially considering the computational cost for large citing sets. Comparing the base and weighted measures, the three originality measures indicate similar levels of positive correlation at $t=1$. While the base measure shows rather stable correlations over time, weighted measure 1 has an increase in the first few years and weighted measure 2 has a

${ }^{3}$ See Online Supplement for the distribution of the measures. 

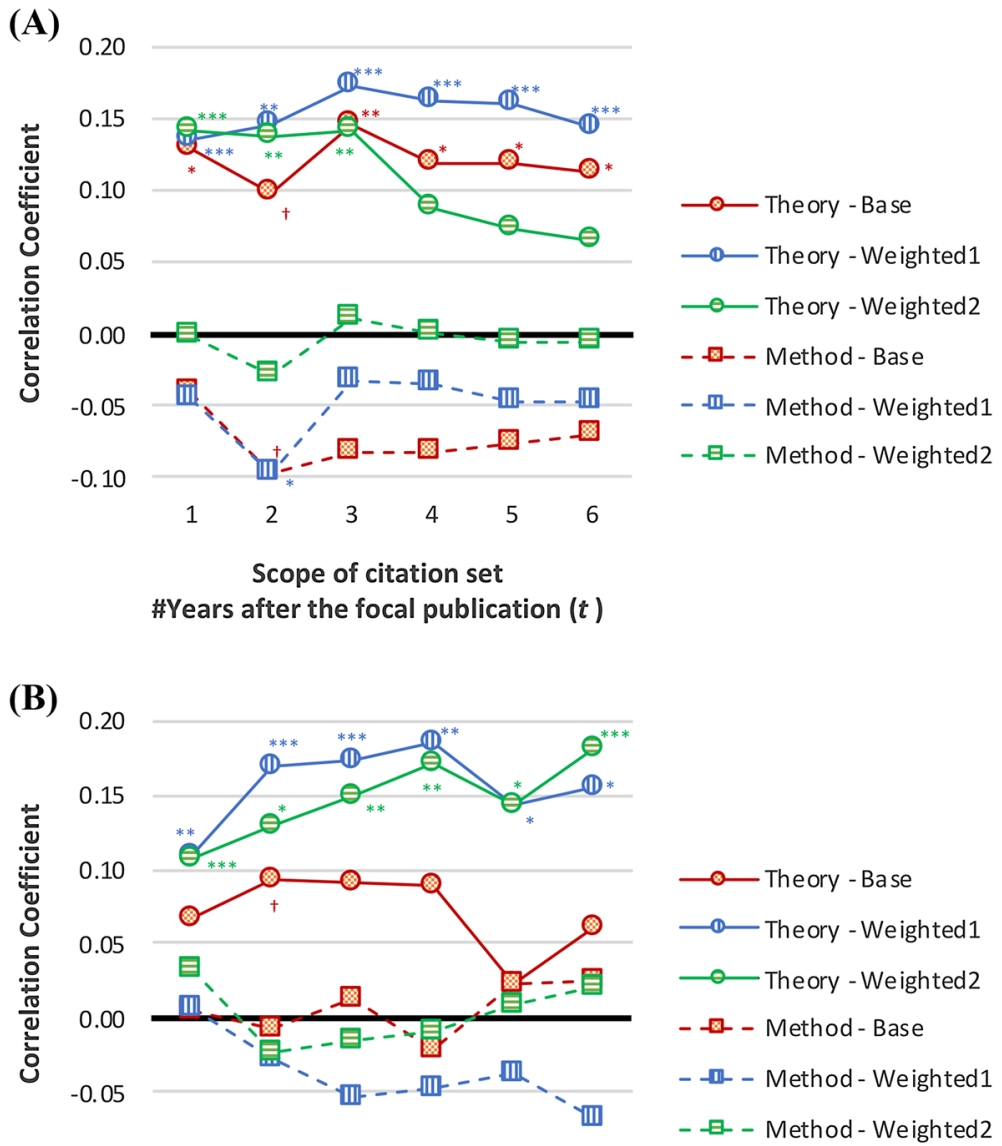

Fig. 2 Correlation between the proposed originality measures and self-assessed originality. Note: $\dagger p<0.1$; ${ }^{*} p<0.05 ; * * p<0.01 ; * * * p<0.001$. A The sample size ranges from 461 to 547 . B The sample size ranges from 354 to 540 . Since our respondents can have multiple papers during their PhD study, we introduced a weight (the reciprocal of the paper count) into the computation of correlation coefficients. See Online Supplement for the correlation analyses

decrease after the third year. Figure 3 a illustrates the joint distribution of the self-assessed measure and the originality measures based on the first-year citing papers.

Next, we alternatively focus on the first $s$ citing papers to calculate our originality measures $(s=1, \ldots, 6)$. Figure $2 \mathrm{~b}$ confirms significant correlations with theoretical originality (for example, $r_{\text {base }}=0.093, p<0.1 ; r_{\text {weighted } 1}=0.169, p<0.001 ; r_{\text {weighted } 2}=0.130, p<0.05$ at $s=2)$ but not with methodological originality $(p>0.1)$, though the correlation with the base measure becomes mostly insignificant. The result also suggests that only the first few citing papers contribute to the positive correlation with theoretical originality and that 


\section{(A) Originality computed with first-year citing papers $(t=1)$}
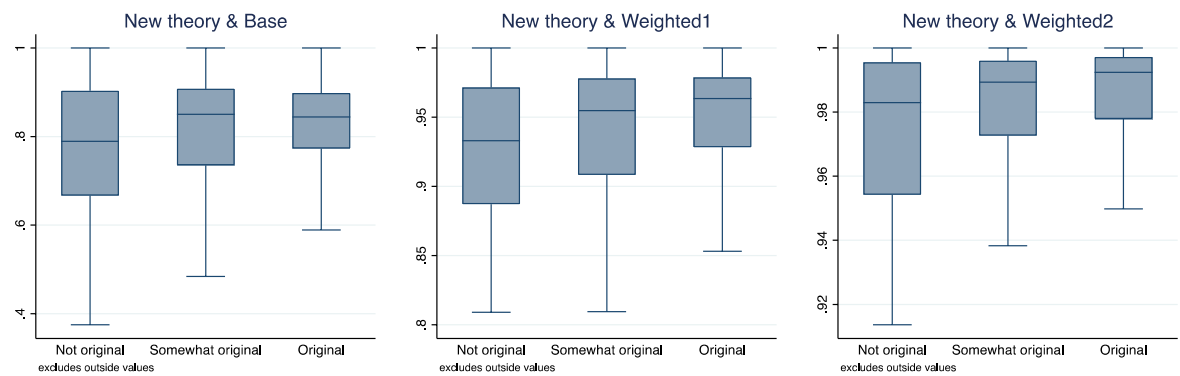

(B) Originality computed with the first three citing papers $(s=3)$
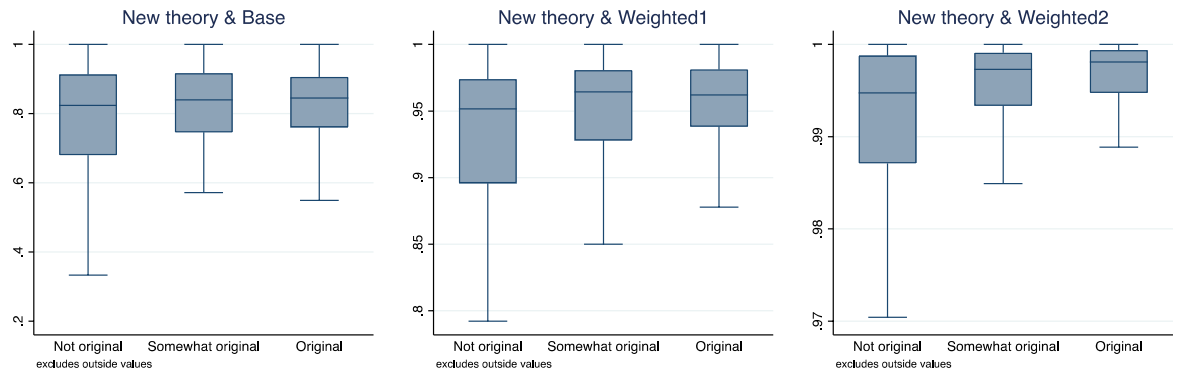

Fig. 3 Joint distribution of self-assessed originality and proposed originality measures

including more citing papers does not improve the correlation. Figure $3 \mathrm{~b}$ shows the joint distribution of the self-assessed measure and the originality measure.

For the respondents who have multiple papers during their PhD study, we also took the mean of the originality measures for each respondent, and analysed the correlation at the scientist level instead of the paper level. This approach tends to present higher correlation coefficients (See Online Supplement), probably because taking the mean mitigates potential volatility of the originality measures at the paper level.

These results imply that the originality measures can be calculated with a small number of citing papers published shortly after the focal paper. That is, reliable measurement is feasible without needing to wait for a long time and with limited computational cost, lending practical utility to the proposed measures.

\section{Prediction of future citation}

We next test whether the proposed measures can predict the citation impact in the future. We compute the originality measures based on the first-year citing papers as the independent variable and predict whether the focal paper becomes among the top $10 \%$ highly-cited using citation count up to 2018. The regression model is specified as

$$
\operatorname{Pr}(\operatorname{Top} 10=1)=f\left(\beta_{0}+\beta_{1} \text { Orig }+\beta_{2} \ln N+\beta_{3} \ln M+\mu+\varepsilon\right)
$$


Table 1 Prediction of citation rank

(A) Originality measured with first-year citing papers

\begin{tabular}{lllllll}
\hline & Model 1 & \multicolumn{3}{c}{ Model 2 3} \\
\hline $\ln \boldsymbol{M}$ & $4.157^{* * *}$ & $(0.813)$ & $3.597^{* * *}$ & $(0.781)$ & $3.648^{* * *}$ & $(0.803)$ \\
$\ln \boldsymbol{N}$ & 0.504 & $(0.745)$ & 0.577 & $(0.692)$ & 0.587 & $(0.680)$ \\
Orig $_{\text {base }}$ & $9.316^{* *}$ & $(3.108)$ & & & & \\
Orig $_{\text {weighted } 1}$ & & & $13.167^{\dagger}$ & $(7.192)$ & & \\
Orig $_{\text {weighted } 2}$ & & & & & $35.700^{*}$ & $(15.480)$ \\
$\chi^{2}$ statistic & $32.574 * * *$ & & $23.885^{* * *}$ & & $24.417^{* * *}$ & \\
Log likelihood & -102.924 & & -104.609 & & -103.633 & \\
$N$ & 399 & & 399 & & 399 & \\
\#Scientists & 192 & & 192 & & 192 & \\
\hline & & & & & &
\end{tabular}

(B) Originality measured with $s$ citing papers $(s=2,3$, and 4)

\begin{tabular}{lllllll}
\hline & $s=2$ & \multicolumn{3}{c}{$s=4$} & \\
\hline Orig $_{\text {base }}$ & 1.149 & $(1.553)$ & $4.442^{*}$ & $(2.116)$ & $5.313^{*}$ & $(2.349)$ \\
Orig $_{\text {weighted1 }}$ & 2.780 & $(3.795)$ & $10.910^{*}$ & $(5.144)$ & $14.686^{* *}$ & $(5.266)$ \\
Orig $_{\text {weighted2 }}$ & $107.669 * * *$ & $(31.184)$ & $162.262^{* * *}$ & $(45.624)$ & $166.365^{* * *}$ & $(48.996)$ \\
$N$ & 439 & & 410 & & 375 & \\
\#Scientists & 205 & & 194 & & 185 & \\
\hline
\end{tabular}

Logistic regressions with errors clustered in scientists. Unstandardised coefficients (robust errors in parentheses). Two-tailed test. $\dagger p<0.1 ;{ }^{*} p<0.05 ; * * p<0.01$; $* * * p<0.001$. Papers in 2008 or before are included as focal papers in the analysis to allow a 10-year citation window. (B) Only the coefficients of the originality measures are presented. The complete regression tables are presented in Online Supplement

As the dependent variable is binary, we use a logit regression model, and $f$ is the logistic function. As a scientist can have multiple papers in the sample, we control for random errors at the scientist level $(\mu)$. Finally, we control for the log number of references $(N)$ and citations in the first year $(M)$. The model prediction is based on the maximum likelihood estimation. Here we focus on focal papers before 2008 so that the time window for accumulating citations is at least 10 years.

Table 1A presents the result of the analyses, finding significantly positive coefficients: $b_{\text {base }}=9.316, p<0.01$ (Model 1); $b_{\text {weighted } 1}=13.167, p<0.1$ (Model 2); $b_{\text {weighted } 2}=35.700$, $p<0.05$ (Model 3). Figure 4a graphically illustrates the result, suggesting that papers with higher originality scores are significantly more likely to be highly cited in the future. Noticeably, the citation count in the first year $(M)$ significantly correlates with both the dependent variable and the originality measures, which can confound the analysis. Thus, we test the predicting power of the originality measures with a fixed number of citing papers, by using the sub-sample of papers that have at least $s$ citations in the first three year $(s=2,3$, and 4$)$. Table $1 \mathrm{~B}$ summarises the results, suggesting that the originality measures computed only with a few citing papers can reasonably predict future citations. Figure $4 \mathrm{~b}$ graphically presents the result, suggesting that focal papers with higher originality scores are significantly more likely to be highly cited. Because we expect a positive association between originality and future citations, this finding demonstrates the construct validity of our originality measure (Babbie 2012). 
(A) Originality computed with first-year citing papers $(t=1)$
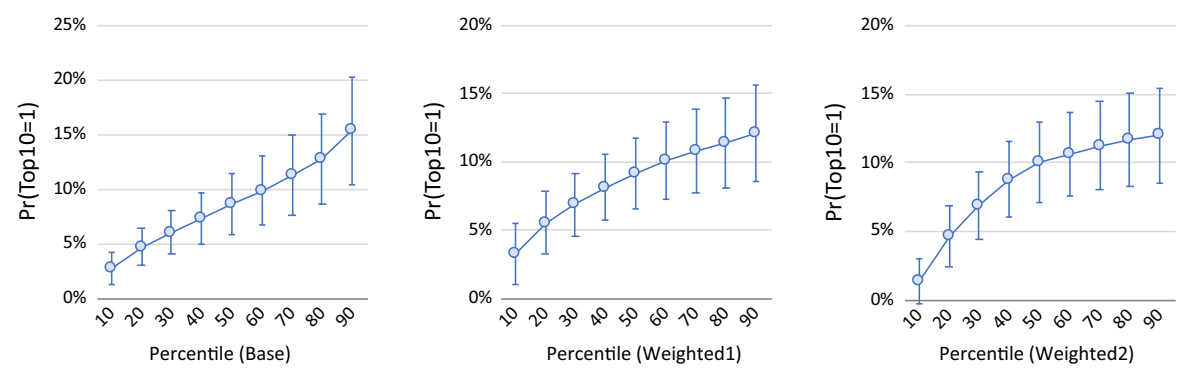

(B) Originality computed with the first three citing papers $(s=3)$
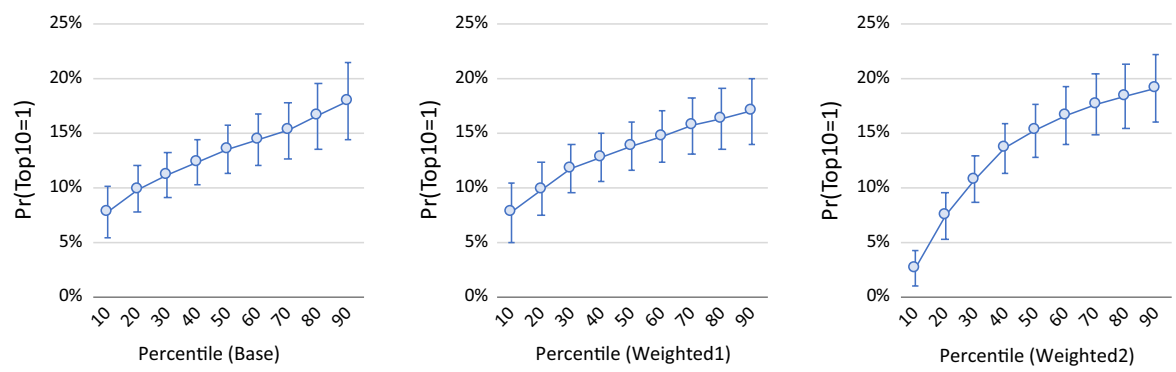

Fig. 4 Prediction of citation rank. Note: The probability of a focal paper falling within top 10 percentile is predicted on the basis of regression models (Table 1). To facilitate interpretation, the horizontal axis takes the percentile of the originality measures. Error bars indicate one standard error

\section{Characterisation of originality measures}

We further investigate the behaviour of the proposed originality measures. Specifically, we examine the following ideas. First, since the above analyses suggest that citing papers in later time horizon have little added value for measuring originality, we test to what extent remote citing papers are relevant for measuring originality. Second, the proposed measures are positively correlated with the citation count and the reference count of the focal article, so we aim to confirm that the proposed measures do capture the originality of focal papers even after these confounding factors are controlled. Third, since any bibliometric indicator can be biased by contextual factors, we test particularly whether the publication year and the subfields of focal papers influence the measures.

To these ends, we compute a series of originality measures (Orig) based on citing papers in $t$-th year following the publication year of the focal paper $(t=1, \ldots, 10)$. Here, the citing set includes papers in the specific one year (but not up to that year). We regress this series of originality scores on the self-assessed originality measure, as well as other confounding factors (Table 2). The regression model is specified as

$$
\text { Orig }=\beta_{0}+\boldsymbol{D}^{T} \boldsymbol{\beta}_{1}+\text { OrigTheory } \boldsymbol{D}^{T} \boldsymbol{\beta}_{2}+\beta_{3} \ln N+\beta_{4} \ln M+\text { Field }^{T} \boldsymbol{\beta}_{5}+\text { PubYear }^{T} \boldsymbol{\beta}_{6}+\mu+v+\varepsilon
$$

where $\boldsymbol{D}^{T}$ is a row vector of time dummies with the $t$-th entry $d_{t}=1$ and other entries $=0$; OrigTheory is the self-assessed originality measure; $N$ is the number of references; $M$ is the number of citing papers in the citing set; Field $^{T}$ is a row vector of field dummies; 
Table 2 Prediction of originality

\begin{tabular}{|c|c|c|c|c|c|c|}
\hline \multirow{3}{*}{$\overline{\ln M}$} & \multirow{2}{*}{\multicolumn{2}{|c|}{$\frac{\text { Model } 1}{\text { Orig }}$}} & \multirow{2}{*}{\multicolumn{2}{|c|}{$\frac{\text { Model } 2}{\text { Orig }_{\text {weighted } 1}}$}} & \multirow{2}{*}{\multicolumn{2}{|c|}{$\frac{\text { Model } 3}{\text { Orig }_{\text {weighted2 }}}$}} \\
\hline & & & & & & \\
\hline & .001 & $(.003)$ & $.006^{* * * *}$ & $(.001)$ & $.002 *$ & $(.001)$ \\
\hline $\ln N$ & $.074 * * *$ & $(.007)$ & $.047 * * *$ & $(.004)$ & $.023 * * *$ & $(.004)$ \\
\hline \multicolumn{7}{|l|}{$d_{1}$ (base group) } \\
\hline$d_{2}$ & $.057 * * *$ & $(.013)$ & $.021 * * *$ & $(.006)$ & $.030 * * *$ & $(.004)$ \\
\hline$d_{3}$ & $.055 * * *$ & $(.013)$ & $.025 * * *$ & $(.006)$ & $.034 * * *$ & $(.004)$ \\
\hline$d_{4}$ & $.094 * * *$ & $(.014)$ & $.037 * * *$ & $(.006)$ & $.040 * * *$ & $(.004)$ \\
\hline$d_{5}$ & $.089 * * *$ & $(.014)$ & $.038 * * *$ & $(.007)$ & $.042 * * *$ & $(.004)$ \\
\hline$d_{6}$ & $.118 * * *$ & $(.013)$ & $.049 * * *$ & $(.006)$ & $.045 * * *$ & $(.004)$ \\
\hline$d_{7}$ & $.131 * * *$ & $(.014)$ & $.056^{* * *}$ & $(.007)$ & $.044 * * *$ & $(.004)$ \\
\hline$d_{8}$ & $.126^{* * * *}$ & $(.014)$ & $.053 * * *$ & $(.007)$ & $.043 * * *$ & $(.004)$ \\
\hline$d_{9}$ & $.125 * * *$ & $(.014)$ & $.057 * * *$ & $(.007)$ & $.045^{* * *}$ & $(.004)$ \\
\hline$d_{10}$ & $.135 * * *$ & $(.014)$ & $.059 * * *$ & $(.007)$ & $.044 * * *$ & $(.004)$ \\
\hline OrigTheory $\times d_{1}$ & $.015^{\dagger}$ & $(.008)$ & $.011^{*}$ & $(.004)$ & $.013 * * *$ & $(.004)$ \\
\hline OrigTheory $\times d_{2}$ & -.003 & $(.008)$ & .007 & $(.004)$ & .005 & $(.004)$ \\
\hline OrigTheory $\times d_{3}$ & .014 & $(.008)$ & $.011^{*}$ & $(.005)$ & .005 & $(.004)$ \\
\hline OrigTheory $\times d_{4}$ & -.003 & $(.009)$ & .007 & $(.005)$ & .003 & $(.004)$ \\
\hline OrigTheory $\times d_{5}$ & .006 & $(.009)$ & $.008^{\dagger}$ & $(.005)$ & .002 & $(.004)$ \\
\hline OrigTheory $\times d_{6}$ & -.004 & $(.009)$ & .003 & $(.005)$ & .001 & $(.004)$ \\
\hline OrigTheory $\times d_{7}$ & -.009 & $(.009)$ & -.000 & $(.005)$ & .002 & $(.004)$ \\
\hline OrigTheory $\times d_{8}$ & -.007 & $(.009)$ & .002 & $(.005)$ & .002 & $(.004)$ \\
\hline OrigTheory $\times d_{9}$ & .000 & $(.009)$ & .003 & $(.005)$ & .002 & $(.004)$ \\
\hline OrigTheory $\times d_{10}$ & -.004 & $(.009)$ & .002 & $(.005)$ & .002 & $(.004)$ \\
\hline Field dummies & Yes** & & Yes & & Yes & \\
\hline PubYear dummies & Yes & & Yes & & Yes & \\
\hline$\chi^{2}$ statistic & $751.245 * * *$ & & $615.091 * * *$ & & $613.655^{* * * *}$ & \\
\hline Log likelihood & 3399.564 & & 6002.593 & & 7859.201 & \\
\hline$N$ & 3641 & & 3641 & & 3641 & \\
\hline \#Papers & 486 & & 486 & & 486 & \\
\hline \#Scientists & 214 & & 214 & & 214 & \\
\hline
\end{tabular}

Unstandardised coefficients (robust errors in parentheses). Random errors are clustered in scientists and in papers. Two-tailed test. ${ }^{\dagger} p<0.1 ;{ }^{*} p<0.05$; $* * p<0.01 ; * * *<<0.001$. The statistical significance of the field dummies and that of publication-year dummies are jointly tested. Papers in 2008 or before are included as focal papers in the analysis to allow a 10-year citation window

PubYear $^{T}$ is a row vector of publication-year dummies; $\mu$ is the random error at the scientist level; and $v$ is the random error at the paper level. The model prediction is based on the maximum likelihood estimation.

First, the result finds that the time dummies $\left(d_{1}, \ldots, d_{10}\right)$ have significantly positive coefficients $(p<0.001)$ and their magnitude increases over time. This implies that the proposed originality measure increases over time when we include citing papers that is timewise more distant from the focal paper. This is probably because citing papers generally deviate 
from the focal study over time, and this implies that using long time windows for assessing originality could cause errors rather than to add information.

Second, the models include the interaction terms between the time dummies and the self-assessed originality measure (OrigTheory) to evaluate the temporal dynamics in the correlation between the self-assessed originality and the proposed originality measures. Each interaction term is concerned with to what extent the citing papers in the particular year capture the originality of the focal paper. Consistent with the above analyses, the result shows that the coefficients of the interaction terms are significant only in the first few years (base: $t=1$ (Model 1), weighted 1: $t=1,3,5$ (Model 2), weighted 2: $t=1$ (Model 3)). This suggests that citing papers in later time horizon do not provide additional information about originality.

Third, as expected, both citation count $(M)$ and the number of references $(N)$ are positively correlated with the originality measures. Even after controlling for them, the proposed measures are significantly positively correlated with the self-assessed originality measure. Thus, the proposed measures seem to capture the true originality.

Fourth, the models include series of dummy variables for publication years (PubYear) and scientific subfields, and the result finds their negligible effects on the proposed measures except for the base measure (Model 1). Thus, at least within the scope of our sample, the contextual difference of the proposed measures seems limited.

\section{Discussion}

The results highlight several features of the proposed measure. First, it presents a significant correlation with scientists' self-assessment of originality. In particular, the proposed measure is correlated with theoretical newness (but not with methodological newness), which has previously been found as the main source of originality in life sciences (Dirk 1999). Because of multi-dimensionality of originality (Guetzkow et al. 2004), it is crucial to understand what aspect of originality is captured by any bibliometric indicator. Second, the operationalisation of our proposed measure is consistent with the betweenness centrality in a directed network (Borgatti and Everett 2006; Freeman 1979). Betweenness centrality has already been actively used in bibliometric studies, but prior studies have rarely analysed directed citation network to identify the flow of knowledge. Third, our measure builds not only on references but also on forward citations and therefore can be manipulated to a lesser extent by authors. Because of this advantage, our measure provides a robust tool for studying science as well as for research evaluation and science decision-making. Fourth, our proposed measure requires a smaller computational cost, especially when compared with the previous novelty measures that require information about the whole universe of papers. Our results suggest that the proposed measure can be computed with limited scope of citation network and without needing to wait for a long time after publication. This adds to the practical utility of our proposed measure. Fifth, our proposed measure helps predict future citation impact. Original discoveries are supposed to be the source of scientific progress, and the result shows that papers with a higher level of originality are also more likely to be highly cited in the long run. This adds to the validity of the proposed measure. Sixth, this study characterises the detailed behaviour of the measure, including its temporal dynamics and contextual contingencies. The result offers guidance for using the measures in future research. 
Our approach has a few limitations and further research is needed. First, although we assume that citation links embody the flow of knowledge, citations can be made for various reasons (Bornmann and Daniel 2008; Martin and Irvine 1983; Wang 2014), which challenges the validity of our measure. Second, the proposed measure can be computed only if a citation is made. In addition certain types of original discoveries may be recognised only long after their publication (e.g., sleeping beauties) (Van Raan 2004), which cannot be captured by our measure based on short-time citations. Third, there are important differences between disciplines in citation behaviour, but our validation is limited to the field of life sciences. The field is known to have the fastest citation accumulation process compared with other disciplines (Wang 2013), which allows us to compute the originality measure in a short time window, but other fields might need longer citation time windows. Future research should identify the optional citing sets for different fields. Fourth, as self-reported originality measures can be biased, the proposed measure could be further validated by alternative approaches such as the use of scientific awards (e.g., Nobel prize) and a text analysis to detect languages associated with originality.

In conclusion, this study proposes a new bibliometric measure of originality. Although originality is a core value in science (Dasgupta and David 1994; Merton 1973; Stephan 1996; Storer 1966), measuring originality in a large scale has been a formidable challenge. Our proposed measure builds on the network betweenness centrality concept (Borgatti and Everett 2006; Freeman 1979) and demonstrates several favourable features as discussed above. We expect that the proposed measure offers an effective tool not only for scholarly research on science but also for practices in research evaluation and various science decision-makings.

Acknowledgments Open access funding provided by Lund University. This study was financially supported by the Japan Society for the Promotion of Science (16K01235).

Open Access This article is distributed under the terms of the Creative Commons Attribution 4.0 International License (http://creativecommons.org/licenses/by/4.0/), which permits unrestricted use, distribution, and reproduction in any medium, provided you give appropriate credit to the original author(s) and the source, provide a link to the Creative Commons license, and indicate if changes were made.

\section{Appendix 1}

We claim that the proposed measure in Eq. (3) is equivalent to the normalised betweenness centrality of $X$ in the directed network, in which the focal paper $X$, papers in citing set $C$, and papers in reference set $R$ are the nodes, and citation links between them are directional edges. The normalised betweenness centrality of node $\mathrm{X}$ is defined as

$$
\frac{1}{S} \sum_{n \neq X \neq m} \frac{\sigma_{n m}(X)}{\sigma_{n m}}
$$

where $\sigma_{n m}(X)$ is the number of paths from node $n$ to node $m$ that are shortest and go through node $X, \sigma_{n m}$ is the number of paths from node $n$ to node $m$ that are shortest, and $S$ is a normalisation factor: the number of all possible paths among all nodes in the network (Freeman 1979).

In the given network, the shortest path between $n$ and $m$ is either a direct citation link from $m$ to $n$ or an indirect citation link through $X$. In either case, the shortest path is unique. Thus, $\sigma_{n m}=1 \forall n, m$. If $m$ cites $n$ (i.e., $x_{n m}=1$ ), $n$ and $m$ are directly linked, and thus, $\sigma_{n m}(X)=0$. If $m$ does not cite $n$ (i.e., $x_{n m}=0$ ), then $n$ and $m$ are linked only through $X$, and thus, $\sigma_{n m}(X)=1$. Thus, $\sigma_{n m}(X)=1-x_{n m}$. 
In directed networks, the number of possible paths is given as $S=\left(l_{I}-1\right)\left(l_{O}-1\right)$, where $l_{I}$ is the number of nodes with incoming links and $l_{O}$ is the number of nodes with outgoing links (White and Borgatti 1994). In the given network, $l_{I}=M+1$ and $l_{O}=N+1$. Therefore,

$$
\frac{1}{S} \sum_{n \neq X \neq m} \frac{\sigma_{n m}(X)}{\sigma_{n m}}=\frac{1}{(M+1-1)(N+1-1)} \sum_{n \neq X \neq m} \frac{1-x_{n m}}{1}=1-\frac{1}{M N} \sum_{m=1}^{M} \sum_{n=1}^{N} x_{n m}
$$

\section{Appendix 2}

To address potential biases in the originality measure in Eq. (3), we propose weighted measures in Eqs. (4) and (5) that control for the reference count of each paper in citing set $C$ and the citation count of each paper in reference set $R$.

Suppose that papers choose their references from the universe of all existing papers with different probabilities (Barabasi and Albert 1999). Each paper in the universe has different visibility or the likelihood of being cited, which we denote by $f(\cdot)$-a non-decreasing function of the citation count of the paper. Focus on the citations between the $n$th reference in $R$ and the $m$-th citing paper in $C$. Suppose that the $m$-th citing paper chooses one reference from the universe of papers. The probability of the $n$-th reference being cited is given by $f\left(z_{n}\right)$. Since the $m$-th citing paper has $y_{m}$ references, the probability of the $n$-th reference being one of the references is given by $1-\left(1-f\left(z_{n}\right)\right)^{y_{m}} \cong y_{m} f\left(z_{n}\right)$, where the approximation holds because $f\left(z_{n}\right) \ll 1$. Summing this up across all combinations of references and citing papers, the expected number of citations between $R$ and $C, E\left(\sum_{m=1}^{M} \sum_{n=1}^{N} x_{n m}\right)$, is given by $\sum_{m=1}^{M} y_{m} \sum_{n=1}^{N} f\left(z_{n}\right)$. In Eq. (3), we use $M N$ to normalise $\sum_{m=1}^{M} \sum_{n=1}^{N} x_{n m}$. An alternative normalisation factor is the expected value of $\sum_{m=1}^{M} \sum_{n=1}^{N} x_{n m}$. Hence,

$$
1-\frac{\sum_{m=1}^{M} \sum_{n=1}^{N} x_{n m}}{E\left(\sum_{m=1}^{M} \sum_{n=1}^{N} x_{n m}\right)}=1-\frac{\sum_{m=1}^{M} \sum_{n=1}^{N} x_{n m}}{\sum_{m=1}^{M} y_{m} \sum_{n=1}^{N} f\left(z_{n}\right)}
$$

In particular, we employ two forms of $f(\cdot)$. For simplicity, we first assume that $f(\cdot)$ is a constant function: $f\left(z_{n}\right)=1 / L$, which gives the weighted measure in Eq. (4). Here, $L$ is an adjusting factor such that the summation of $f(\cdot)$ across the universe of papers equals 1 , or the number of the papers in the universe. Second, following the prior literature (Barabasi and Albert 1999), we assume that $f(\cdot)$ is proportionate to the citation count of each paper: $f\left(z_{n}\right)=z_{n} / L$, where $L$ is the total number of forward citations that exist in the paper universe. This gives the second weighted measure in Eq. (5). Though $L$ is unknown, we assume that $L$ is constant across our sample papers. To facilitate interpretation, we set $L$ such that the minimum value of originality scores is zero. 


\section{Appendix 3}

Description of self-assessed originality measures. Note: A $N=236$. B $N=234$.

(A) Theoretical originality

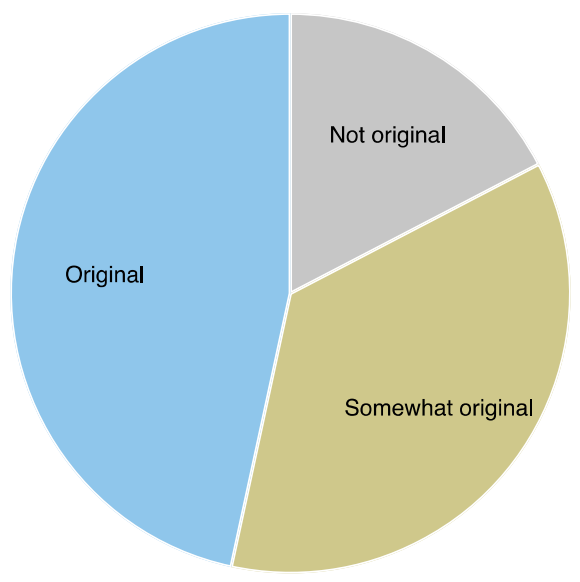

(B) Methodological originality

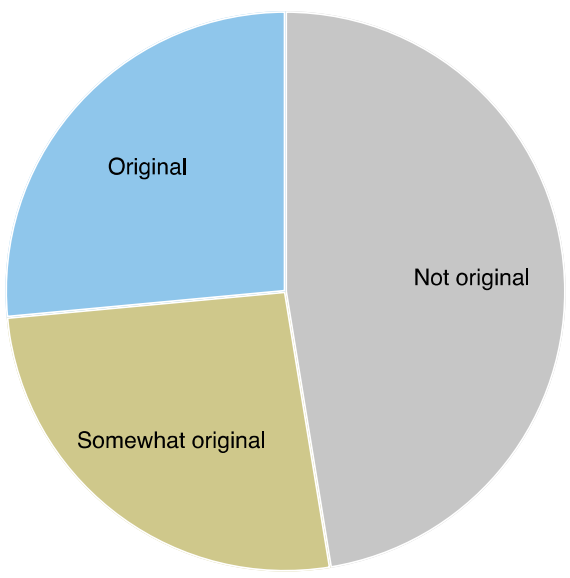

\section{References}

Amabile, T. M. (1983). The social psychnology of creativity: A componential conceptualization. Journal of Personality and Social Psychology, 45, 357-376.

Azoulay, P., Zivin, J. S. G., \& Manso, G. (2011). Incentives and creativity: Evidence from the academic life sciences. Rand Journal of Economics, 42, 527-554.

Babbie, E. R. (2012). The practice of social research. Belmont, CA: Wadsworth Publishing.

Barabasi, A. L., \& Albert, R. (1999). Emergence of scaling in random networks. Science, 286, 509-512.

Borgatti, S. P., \& Everett, M. G. (2006). A graph-theoretic perspective on centrality. Social Networks, 28, 466-484.

Bornmann, L., \& Daniel, H. D. (2008). What do citation counts measure? A review of studies on citing behavior. Journal of Documentation, 64, 45-80.

Boudreau, K. J., Guinan, E. C., Lakhani, K. R., \& Riedl, C. (2016). Looking across and looking beyond the knowledge frontier: Intellectual distance, novelty, and resource allocation in science. Management Science, 62, 2765-2783.

Bourdieu, P. (1975). The specificity of the scientific field and the social conditions for the progress of reason. Social Science Information, 14, 19-47.

Brandes, U. (2008). On variants of shortest-path betweenness centrality and their generic computation. Social Networks, 30, 136-145.

$\mathrm{Bu}$, Y., Waltman, L., Huang, Y. 2019. A multidimensional perspective on the citation impact of scientific publications. arXiv preprint arXiv:1901.09663.

Chubin, D. E., \& Hackett, E. J. (1990). Peerless science: Peer review and U.S. science policy. Albany, NY: State University of New York Press.

Dasgupta, P., \& David, P. A. (1994). Toward a new economics of science. Research Policy, 23, 487-521.

De Bellis, N. (2009). Bibliometrics and citation analysis: From the science citation index to cybermetrics. Lanham, MD: Scarecrow Press.

Dirk, L. (1999). A measure of originality: The elements of science. Social Studies of Science, 29, 765-776.

Ercsey-Ravasz, M., Lichtenwalter, R. N., Chawla, N. V., \& Toroczkai, Z. (2012). Range-limited centrality measures in complex networks. Physical Review E, 85, 066103. 
Flynn, F. J., \& Wiltermuth, S. S. (2010). Who's with me? False consensus, brokerage, and ethical decision making in organizations. Academy of Management Journal, 53, 1074-1089.

Foster, J. G., Rzhetsky, A., \& Evans, J. A. (2015). Tradition and innovation in scientists' research strategies. American Sociological Review, 80, 875-908.

Freeman, L. C. (1979). Centrality in social networks conceptual clarification. Social Networks, 1, $215-239$.

Freeman, L. C., Borgatti, S. P., \& White, D. R. (1991). Centrality in values graphs-A measure of betweenness based on network flow. Social Networks, 13, 141-154.

Funk, R. J., \& Owen-Smith, J. (2017). A dynamic network measure of technological change. Management Science, 63, 791-817.

Garfield, E. (1955). Citation indexes for science-New dimension in documentation through association of ideas. Science, 122, 108-111.

Gaston, J. C. (1973). Originality and competition in science. Chicago, IL: University of Chicago Press.

Gilbert, G. N. (1977). Referencing as persuasion. Social Studies of Science, 7, 113-122.

Glänzel, W., \& Schoepflin, U. (1995). A bibliometric study on ageing and reception processes of scientific literature. Journal of information Science, 21, 37-53.

Gomez, D., Figueira, J. R., \& Eusebio, A. (2013). Modeling centrality measures in social network analysis using bi-criteria network flow optimization problems. European Journal of Operational Research, 226, 354-365.

Gould, R. V., \& Fernandez, R. M. (1989). Structures of mediation: A formal approach to brokerage in transaction networks. Sociological Methodology, 19, 89-126.

Guetzkow, J., Lamont, M., \& Mallard, G. (2004). What is originality in the humanities and the social sciences? American Sociological Review, 69, 190-212.

Hagstrom, W. O. (1974). Competition in science. American Sociological Review, 39, 1-18.

Hall, B. H., Jaffe, A. B., \& Trajtenberg, M. (2001). The NBER patent citation data file: Lessons, insights and methodological tools. NBER Working Paper, 8498.

Harrigan, K. R., Di Guardo, M. C., Marku, E., \& Velez, B. N. (2017). Using a distance measure to operationalise patent originality. Technology Analysis \& Strategic Management, 29, 988-1001.

Hicks, D., Wouters, P., Waltman, L., De Rijcke, S., \& Rafols, I. (2015). The leiden manifesto for research metrics. Nature, 520, 429-431.

Kuhn, T. S. (1970). The structure of scientific revolutions. Chicago, MI: University of Chicago Press.

Latour, B., \& Woolgar, S. (1979). Laboratory life: The social construction of scientific facts. Beverly Hills: Sage Publications.

Lee, Y.-N., Walsh, J. P., \& Wang, J. (2015). Creativity in scientific teams: Unpacking novelty and impact. Research Policy, 44, 684-697.

Leydesdorff, L. (2007). Betweenness centrality as an indicator of the interdisciplinarity of scientific journals. Journal of the American Society for Information Science and Technology, 58, 1303-1319.

Martin, B. R., \& Irvine, J. (1983). Assessing basic research: Some partial indivators of scientific progress in radio astronomy. Research Policy, 12, 61-90.

Merton, R. K. (1973). Sociology of science. Chicago: University of Chicago Press.

Moed, H. F., Burger, W., Frankfort, J., \& Van Raan, A. (1985). The application of bibliometric indicators: Important field- and time-dependent factors to be considered. Scientometrics, 8, 177-203.

Price, D. J. D. (1986). Little science, big science. New York, NY: Columbia University Press.

Puzis, R., Altshuler, Y., Elovici, Y., Bekhor, S., Shiftan, Y., \& Pentland, A. (2013). Augmented betweenness centrality for environmentally aware traffic monitoring in transportation networks. Journal of Intelligent Transportation Systems, 17, 91-105.

Shibata, N., Kajikawa, Y., \& Matsushima, K. (2007). Topological analysis of citation networks to discover the future core articles. Journal of the American Society for Information Science and Technology, 58, $872-882$.

Small, H. G. (1978). Cited documents as concept symbols. Social Studies of Science, 8, 327-340.

Stephan, P. E. (1996). The economics of science. Journal of Economic Literature, 34, 1199-1235.

Stirling, A. (2007). A general framework for analysing diversity in science, technology and society. Journal of the Royal Society, Interface, 4, 707-719.

Storer, N. (1966). The social system of science. New York, NY: Holt, Rinehart and Winston.

Topirceanu, A., Udrescu, M., \& Marculescu, R. (2018). Weighted betweenness preferential attachment: A new mechanism explaining social network formation and evolution. Scientific Reports, 8, 14.

Trajtenberg, M., Henderson, R., \& Jaffe, A. (1997). University versus corporate patents: A window on the basicness of invention. Economics of Innovation and New Technology, 5, 19-50.

Trapido, D. (2015). How novelty in knowledge earns recognition: The role of consistent identities. Research Policy, 44, 1488-1500. 
Uzzi, B., Mukherjee, S., Stringer, M., \& Jones, B. (2013). Atypical combinations and scientific impact. Science, 342, 468-472.

Van Raan, A. (2004). Sleeping beauties in science. Scientometrics, 59, 467-472.

Wang, J. (2013). Citation time window choice for research impact evaluation. Scientometrics, 94, 851-872.

Wang, J. (2014). Unpacking the matthew effect in citations. Journal of Informetrics, 8, 329-339.

Wang, J. (2016). Knowledge creation in collaboration networks: Effects of tie configuration. Research Policy, 45, 68-80.

Wang, J., Thijs, B., \& Glänzel, W. (2015). Interdisciplinarity and impact: Distinct effects of variety, balance, and disparity. PLOS ONE, 10, e0127298.

Wang, J., Veugelers, R., \& Stephan, P. E. (2017). Bias against novelty in science: A cautionary tale for users of bibliometric indicators. Research Policy, 46, 1416-1436.

White, D. R., \& Borgatti, S. P. (1994). Betweenness centrality measures for directed graphs. Social Networks, 16, 335-346.

Whitley, R. (1984). The intellectual and social organization of the sciences. Oxford: Oxford University Press.

Wu, L., Wang, D., \& Evans, J. A. (2019). Large teams develop and small teams disrupt science and technology. Nature, 566, 378-382.

Yegros-Yegros, A., Rafols, I., \& D'este, P. (2015). Does interdisciplinary research lead to higher citation impact? The different effect of proximal and distal interdisciplinarity. PLoS ONE, 10, e0135095. 